\title{
Control of Hybrid Systems via Dehybridization
}

\author{
B. Sedghi, B. Srinivasan, R. Longchamp \\ Laboratoire d'Automatique, Ecole Polytechnique Fédérale de Lausanne \\ CH-1015 Lausanne \\ e-mail:babak.sedghi@epfl.ch
}

\begin{abstract}
This paper presents a controller design framework for hybrid systems based on approximate continuous time models and standard continuous control techniques. The procedure has three steps: (i) designing a switching controller so that the initial hybrid system revisits one of its modes, (ii) applying a dehybridization procedure, related to singular perturbation, to eliminate all discrete states and the fast continuous states, and (iii) designing a controller for the continuous dehybridized system using standard techniques. This methodology is applied in simulation for the set point tracking of a stick-slip inertial drive.
\end{abstract}

\section{Introduction}

Hybrid dynamical systems are those with interaction between continuous and discrete dynamics. Modeling, analysis, control, and optimization of such systems are active areas of research $[2,10,15,11]$. The control design for hybrid systems is challenging and complex, since a combination of the continuous and discrete variables is needed. Several approaches have been studied for the same - e.g., logic-based switching [6], supervisory control [9], game-theoretic control [14], predictive control [1], switched controller [5] and hierarchical control [3].

Most of these approaches are based on the exact behavior of the system and thus are confronted with the complexity of the design concerning the combination of the switching laws and dynamics. On the other hand, the perturbation and approximative approaches, which are less studied in the context of hybrid systems, can be a reasonable alternative for control design.

In this paper, the idea is to approximate a given hybrid system by a continuous time model and then, use a standard continuous control technique for controller design. Towards this end, a model reduction procedure, that is related to the singular perturbation theory [8], is proposed. With such a procedure, all discrete states and the fast continuous states are eliminated. Since the reduced system loses its hybrid nature, the proposed procedure is more than just model reduction and is termed dehybridization. Among the approaches presented in the literature, the technique that comes closest to dehybridization is related to pulse-width modulation as presented in [12].

The pre-requisites for dehybridization are: (i) A hybrid system should have a repetitive mode, i.e., the system revisits one of its discrete states, and (ii) the time interval between two visits, is sufficiently small or one of the modes is dominant. If the initial system does not possess the above characteristic, an additional inner (hybrid) controller is necessary to render the combination with a repetitive mode.

The proposed control methodology is applied to the set-point tracking problem of a stick-slip inertial drive. Stick-slip drives are friction driven systems, a class to which ultrasonic motors belong. A hybrid model for this system with four continuous states and three discrete states is presented. The system by itself does not have a repetitive mode and so, an inner switching controller is designed to render the combination with a repetitive mode. Using the dehybridization approach, all the discrete states and two of the continuous states were eliminated. A continuous control law based on feedback linearization is then used.

The paper is organized as follows: In Section 2, hybrid systems and singularly-perturbed systems are briefly discussed. Dehybridization of hybrid systems with a repetitive mode is discussed in Section 3, while the controller design methodology is described in Section 4. Section 5 considers the application of the proposed methodology to an inertial stick-slip drive and Section 6 concludes the paper.

\section{Preliminaries}

To set the notations, hybrid systems and two-time scale systems will be discussed below.

\subsection{Hybrid Systems}

Hybrid dynamical systems are those systems with interaction between continuous and discrete dynamics and can be defined as follows: 
Definition 1 [2] A hybrid system $\mathcal{H}$ is a collection $\mathcal{H}=(Q, X, U, D, F, R, H)$, where

- $q \in Q=\{1, \ldots, l\} \subset \mathbb{Z}^{+}$are the discrete states;

- $x \in X \subseteq \mathbb{R}^{n}$ are the continuous states;

- $u \in U \subseteq \mathbb{R}^{m}$ are the system inputs;

- $D \subseteq Q \times X \times U$ is the domain of $\mathcal{H}$;

- $F: Q \times X \times U \rightarrow \mathbb{R}^{n}$ is a vector field describing the continuous dynamics;

- $R: Q \times X \times U \rightarrow Q \times X$ describes the discrete dynamics;

- $H: Q \times X \times U \rightarrow Y \subset \mathbb{R}^{p}$ describes the output map.

The evolution of the system states $(q, x)$ and output $y$ can be described by the following relations:

$$
\begin{aligned}
\left(q_{+}, x_{+}\right) & =R\left(q_{-}, x_{-}, u\right), q(0)=q_{0} \\
\dot{x} & =F(q, x, u), x(0)=x_{0} \\
y & =H(q, x, u)
\end{aligned}
$$

where $(.)_{-}$and $(.)_{+}$refer to variables before and after a transition and $x_{0}$ and $q_{0}$ are the initial conditions of the continuous and discrete states, respectively. The discrete state $q$ will also be referred to as mode $q$. The notation $F_{q}(x, u)$ and $H_{q}(x, u)$ will be used instead of $F(q, x, u)$ and $H(q, x, u)$ to stress the fact that the vector field $F$ changes significantly when $q$ changes.

In this paper, the following two assumptions will be made: (i) for every $q$, the vector field $F_{q}$ is analytic in $\mathbb{R}^{n} \times \mathbb{R}^{m}$, and (ii) there are no discontinuities in the states $x$, i.e., $x_{-}=x_{+}$.

\subsection{Singularly-perturbed Systems}

Consider a system that exhibits two-time scale behavior, i.e., a fast dynamics and a slow one as given in:

$$
\dot{x}=\left(\begin{array}{c}
\dot{\xi} \\
\dot{\eta}
\end{array}\right)=\left(\begin{array}{c}
f(\xi, \eta, u, \epsilon) \\
\frac{1}{\epsilon} g(\xi, \eta, u, \epsilon)
\end{array}\right)
$$

where $\xi$ and $\eta$ represent the slow and fast states, respectively. As $\epsilon \rightarrow 0, \eta$ can be approximated by its quasi-steady state $\eta_{q s s}=\phi(\xi, u)$ obtained by solving $g(\xi, \eta, u, 0)=0$. So, the reduced (slow) system is given by:

$$
\dot{\xi}=f(\xi, \phi(\xi, u), u, 0)=\bar{f}(\xi, u)
$$

The basic assumption in singular perturbation theory is that the dynamics of the fast (boundary-layer) system is exponentially stable. The situation considered here is slightly different where the boundary-layer system is only marginally stable. So, the pseudo-steady state corresponds to the average value around which the fast states oscillate. In either case, the approximation error between (4) and (5) is of order $O(\epsilon)$ [8].

\section{Dehybridization of Hybrid Systems with a Repetitive Mode}

In this section, a model reduction similar to singular perturbation will be discussed, which will eliminate both discrete and continuous states that have fast dynamics. Here all discrete states will removed under the assumption that the transition of discrete states is either fast, or practically no transition takes place.

Since all discrete states are removed, this procedure is more than just a model reduction. In this paper, such an approach is termed dehybridization - removing the hybrid nature from the system dynamics. However, dehybridization can only be done for a class of systems as will be discussed below.

\subsection{Hybrid Systems with a repetitive mode}

Definition 2 A hybrid system $\mathcal{H}$ has a repetitive mode if the sequence of discrete transitions starts with mode $q=M$ and for $\forall q(t) \neq M, \exists \bar{t}>t$ such that $q(\bar{t})=M$.

Let the sequence of discrete states be $\left(q_{0} \rightarrow q_{1} \rightarrow\right.$ $\left.\ldots q_{N} \rightarrow\right)$ such that $q_{0}=q_{N}=M$. Denote the time interval between two occurrences of $q=M$ by $T$. Let $\beta_{q_{j}}$ be the fraction of $T$ with $q(t)=q_{j}$, $j=0, . ., N-1$. Since the discrete states, $q_{j}$, in the sequence may not be distinct, define $\alpha_{i}\left(\alpha_{i} \in[0,1]\right.$, $\left.\sum_{i=1}^{l} \alpha_{i}=1\right)$, such that it sums up $\beta_{q_{j}}$ for $q_{j}=i$, i.e. $\left.\alpha_{i}=\sum_{\left(q_{j}=i\right)} \beta_{q_{j}}, \forall i \in\{1,2, \ldots, l\}\right)$. For $i \in Q$ that are not visited during the sequence, $\alpha_{i}=0$.

\subsection{Elimination of Discrete States}

Theorem 1 Consider a hybrid system (1)-(3) with a repetitive mode and the continuous averaged dynamics:

$$
\dot{\bar{x}}=\sum_{i=1}^{l} \alpha_{i} F_{i}(\bar{x}, u)=\mathrm{F}(\bar{x}, u), \bar{x}\left(t_{0}\right)=x_{0}
$$

If $\lim _{\varepsilon \rightarrow 0} F_{i}(x, u)<\infty, \forall i \in Q$, then the error, $\bar{x}-x=$ $O(\varepsilon)$ in one cycle, where $\varepsilon=T \Pi_{i=1}^{l}\left(1-\alpha_{i}\right)$.

Proof: Let $x_{k}$ and $t_{k}$ denote the solution to the hybrid system and the time when system switches to mode $q_{k}$. Then, from the assumption that $F_{i}$ 's are analytic, the expression for $x_{k}$ and its Taylor's series expansion can be written as:

$$
\begin{aligned}
x_{k} & =x_{0}+\sum_{j=0}^{k-1} \int_{t_{j}}^{t_{j+1}} F_{q_{j}}(x, u) d \tau \\
& =x_{0}+T \sum_{j=0}^{k-1} \beta_{q_{j}} F_{q_{j}}+O\left(T^{2}\right)
\end{aligned}
$$

where $F_{q_{j}}$ stands for $F_{q_{j}}\left(x_{0}, u_{0}\right)$. On the other hand, the solution to the continuous dynamics (6) at $t=t_{k}$, 
$\bar{x}_{k}$, and its Taylor's series expansion noting that $t_{k}=$ $t_{0}+T \sum_{j=0}^{k-1} \beta_{q_{j}}$ can be written as:

$$
\begin{aligned}
\bar{x}_{k} & =x_{0}+\int_{t_{0}}^{t_{k}} \sum_{i=1}^{l} \alpha_{i} F_{i}(x, u) d \tau \\
& =x_{0}+T \sum_{j=0}^{k-1} \beta_{q_{j}} \sum_{i=1}^{l} \alpha_{i} F_{i}+O\left(T^{2}\right)
\end{aligned}
$$

Subtracting (7) from (8), $e_{i}=\bar{x}_{i}-x_{i}$, is given by:

$$
e_{k}=T\left(\sum_{j=0}^{k-1} \beta_{q_{j}} \sum_{i=1}^{l} \alpha_{i} F_{i}-\sum_{j=0}^{k-1} \beta_{q_{j}} F_{q_{j}}\right)+O\left(T^{2}\right)
$$

Suppose $\varepsilon=T \Pi_{i=1}^{l}\left(1-\alpha_{i}\right) \rightarrow 0$, then either (i) $T \rightarrow 0$, (ii) $\exists i$, such that $\alpha_{i} \rightarrow 1$. It is quite obvious that if $T \rightarrow 0$ and $F_{i}$ are bounded, then the first term of (9) goes to zero. If $\alpha_{i} \rightarrow 1$, then $\alpha_{j} \rightarrow 0 \forall j \neq i$ and $\beta_{q_{j}} \rightarrow 0 \forall q_{j-1} \neq i$, from which $\sum_{i=1}^{l} \alpha_{i} F_{i}=F_{i}$ and

$$
\sum_{j=0}^{k-1} \beta_{q_{j}}=\sum_{\substack{j=0 \\\left(q_{j}=i\right)}}^{k-1} \beta_{q_{j}}, \quad \sum_{j=0}^{k-1} \beta_{q_{j}} F_{q_{j}}=\sum_{\substack{j=0 \\\left(q_{j}=i\right)}}^{k-1} \beta_{q_{j}} F_{i}
$$

The above relations imply that the first term in (9) is zero as $\varepsilon \rightarrow 0$. From this, it can be concluded that the error is of order $O(\varepsilon)$.

The above theorem states that the dynamics of the hybrid system in each repetition of mode $M$ can be approximated by a convex combination of the dynamics of the modes visited in that cycle. Note that $\alpha_{i}$ 's used in (6) are functions of the states and contain information regarding the switching. Though analytical expressions for $\alpha_{i}(x)$ are not easy to derive, they can be computed from the measurements of time durations spent in each mode.

\subsection{Removal of Fast Continuous States}

Consider $\varepsilon$ as a parameter of the averaged system. There could be certain components in $F$ that are unbounded as $\varepsilon \rightarrow 0$. These can be written as $\frac{g}{\varepsilon}$, which in turn leads to the standard singular-perturbation formulation:

$$
\dot{\bar{x}}=\left(\begin{array}{c}
\dot{\bar{\xi}} \\
\dot{\bar{\eta}}
\end{array}\right)=\mathrm{F}(\bar{x}, u)=\left(\begin{array}{c}
\mathrm{f}(\bar{\xi}, \bar{\eta}, u, \varepsilon) \\
\frac{1}{\varepsilon} \mathrm{g}(\bar{\xi}, \bar{\eta}, u, \varepsilon)
\end{array}\right)
$$

where $\bar{\xi}$ and $\bar{\eta}$ correspond to the slow and fast states, respectively. $\bar{\eta}$ corresponds to those continuous states that react with, or responsible for, the quick transition of the discrete states. As in the singular perturbation approach, $\bar{\eta}$ can be replaced by their quasi-steady state values $\bar{\eta}_{q s s}=\bar{\phi}(\bar{\xi}, u)$ obtained by solving $\mathrm{g}(\bar{\xi}, \bar{\eta}, u, 0)=$ 0 . Then, the averaged reduced dynamics are:

$$
\dot{\bar{\xi}}=\mathrm{f}(\bar{\xi}, \bar{\phi}(\bar{\xi}, u), u, 0)=\overline{\mathrm{f}}(\bar{\xi}, u)
$$

Since by construction, $f$ and $\bar{f}$ are bounded as $\varepsilon \rightarrow 0$, the approximation errors caused by elimination of both discrete and continuous states will still be of order $O(\varepsilon)$.

\section{Controller Design via Dehybridization}

In the previous section, dehybridization of a hybrid system with a repetitive mode into an approximate averaged continuous dynamics was discussed. In this section, it will be explained how dehybridization can be used for controller design.

The approach proposed in this paper consists of the following steps:

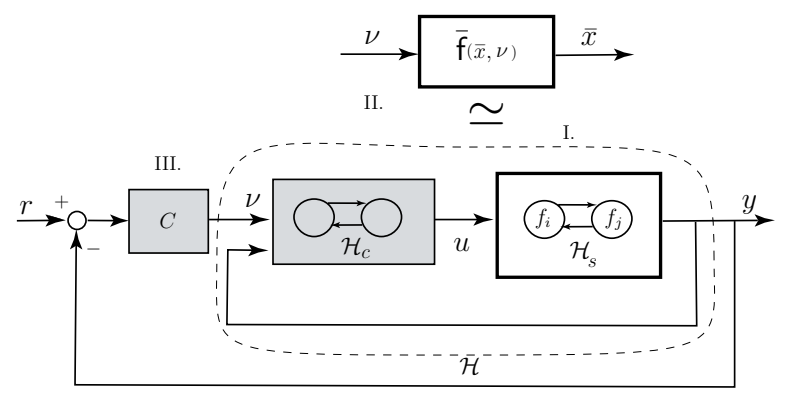

Figure 1: Block diagram for the controller design via dehybridization

I. Rendering the hybrid system with a repetitive mode: For dehybridization to be applied, the hybrid system should have a repetitive mode. However, the initial system $\mathcal{H}_{s}$ need not have one such. So, the first step is to design a switching logic controller, $\mathcal{H}_{c}$, such that the feedback combination of $\mathcal{H}_{c}$ and $\mathcal{H}_{s}$ (as shown in Figure 1 and denoted by $\mathcal{H})$ has a repetitive mode. Note that the goal of $\mathcal{H}_{c}$ is not to stabilize $\mathcal{H}_{s}$ but to only render the combination with a repetitive mode. A systematic procedure to design $\mathcal{H}_{c}$ is not available right now and forms one of the current areas of research. The external input of the controller, $\nu$, takes the task of stabilizing the entire system.

II. Dehybridization of the resulting repetitive hybrid system: As mentioned in Section 3, this step consists of two stages: (i) A continuous averaged model which is a convex combination of the vector fields in each mode is constructed. This way, the discrete states are eliminated. (ii) Using a singular perturbation approach, fast continuous states, that are responsible for, or react with, fast switching between discrete states, are removed. These steps result in an averaged "slow" continuous time system (11).

III. Designing a continuous control for the resulting model: A continuous control law (denoted as $C$ in Figure 1) is designed for (11) using 
standard control techniques such as PID, feedback linearization, or Lyapunov-based designs. Once the stabilizing input $\nu$ is designed for the averaged model, the input for the hybrid system $\mathcal{H}_{s}$ is computed using the inner hybrid controller $\mathcal{H}_{c}$.

The stability is an important issue in the design. For this, ideas from singular perturbation can be extended. If the approximation errors encountered in the averaging process are small enough, then the controller designed for the continuous system will work on the hybrid system also. A detailed analysis will be taken up in the future.

\section{Control of a Stick-Slip Inertial Drive}

\subsection{Hybrid Modeling}

Stick-slip actuators, also referred to as inertial drives, represent a new technology used for positioning in micro-manipulators [4]. A schematic of a stick-slip inertial drive is shown in Figure 2. The advantage of such a system is that the movement range needed by the piezo actuator is much smaller than the movement range of the inertial mass.

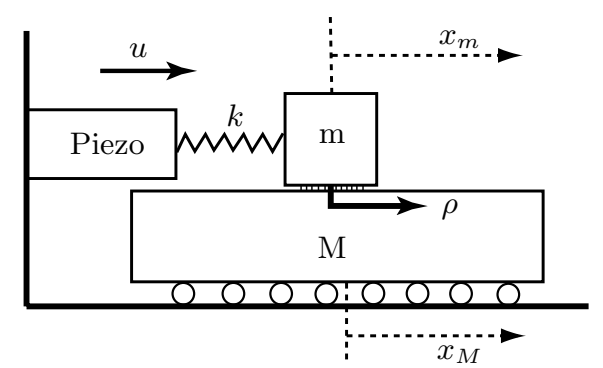

Figure 2: Schematic of Stick-slip Inertial Drive

A simplified model of the stick-slip inertial drive can be developed from first principles:

$$
\begin{aligned}
& \dot{x}_{m}=v_{m}, \quad \dot{v}_{m}=\frac{1}{m}\left(u-k x_{m}-\rho\right) \\
& \dot{x}_{M}=v_{M}, \quad \dot{v}_{M}=\frac{1}{M} \rho
\end{aligned}
$$

where $x_{m}$ and $x_{M}$ are the positions of the small mass and the inertial mass, respectively, with $m$ and $M$ being their respective masses, $u$ the force generated by the piezo actuator, $k$ the spring constant of transmission elements, and $\rho$ the friction force between the two masses. The friction between the inertial mass and the rolling table is neglected. The dynamics of the piezo actuator are much faster than the dynamics of the rest of the system, thus are not considered in this study. The force generated by the piezo actuator (input $u$ ) is limited by the dimension and the load of the system, $|u| \leq u_{\max }$.
The friction $\rho$ is a function of velocity and applied force. Using the two structure friction model [7], the system can be considered as a hybrid system. Depending on the external force and relative velocity, $v_{r}=v_{m}-v_{M}$, three distinct operational modes can be distinguished: if the force, $\mathcal{F}_{i}$, acting at their interface due to an external input, does not exceed the Coulomb friction level, $\mathcal{F}_{c}$, the two masses move together ('stick' mode). As soon as the Coulomb friction level is exceeded, one mass slips over the other. The two modes 'slip $p_{+}$' and 'slip_' are respectively distinguished by positive and negative relative velocities. The discrete states of the system, $q \in\{1,2,3\}$, are defined by three modes $q=1$ (stick), $q=2\left(\operatorname{slip}_{+}\right)$and $q=3\left(\operatorname{slip}_{-}\right)$[13]. The transitions between various modes and friction force $\rho$ are depicted in Figure 3.

The expression for $\mathcal{F}_{i}$ in the 'stick' mode can be derived from the evolution of the relative velocity. Using (12), (13), and $\dot{v}_{m}-\dot{v}_{M}=0$, leads to $\rho=\mathcal{F}_{i}=\frac{M}{m+M}(u-$ $\left.k x_{m}\right)$. In slip $\rho=\mathcal{F}_{c} \operatorname{sgn}\left(v_{r}\right)$.

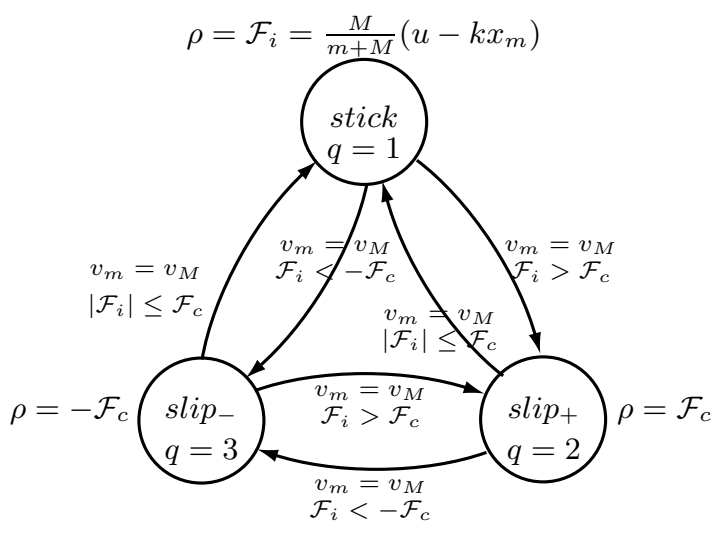

Figure 3: State Transition of $\mathcal{H}_{s}$

\subsection{Design via Dehybridization}

In this section, the dehybridization procedure is used for the set-point tracking problem of the stick-slip inertial drive, i.e., $\lim _{t \rightarrow \infty}\left(r-x_{M}(t)\right) \rightarrow 0$, where $r$ is the set-point.

\section{Rendering the inertial drive with a repet- itive mode}

The stick-slip actuator by itself does not have a repetitive mode. So, the switching logic $\mathcal{H}_{c}$ depicted in Figure 4 is used. Here, $L_{\text {stick }}=\frac{u_{\max }}{k}+\frac{m+M}{k M} \mathcal{F}_{c}$ and $L<L_{\text {stick }}$ is a design parameter. Note that the controller discrete state $q_{c}=1$ is associated with the mode stick of the system, $q_{c}=2,3$ with slip , and $q_{c}=4,5$ with slip $_{+}$. By applying such a logic, the system-controller combination has a repetitive mode.

For example, consider the external input, $\nu$, as shown in Figure 1 to be positive. Also let $v_{m}=v_{M}$, and $x_{m}<L_{\text {stick }}$, so that the system controller combination 


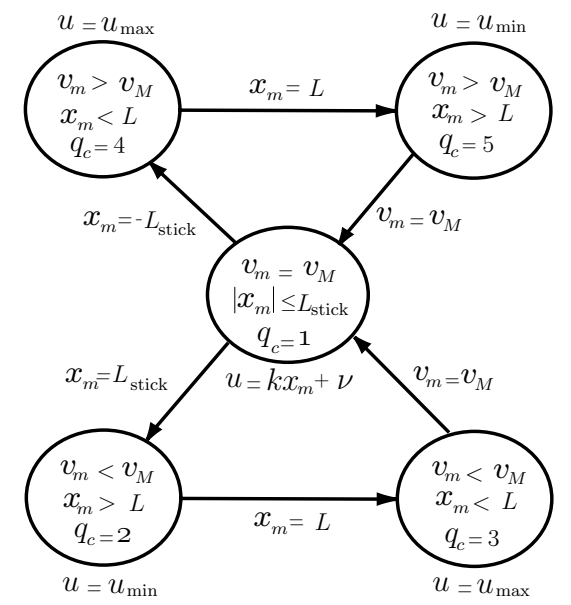

Figure 4: State transition of switching logic $\mathcal{H}_{c}$

will be in the state (stick, $\left.q_{c}=1\right)$ and input $u=$ $k x_{m}+\nu$ is applied. Since $\nu>0, x_{m}$ will increase. When $x_{m}=L_{\text {stick }}$, there is no feasible input for the system to continue in the stick mode. So it will switch to (slip , $q_{c}=2$ ), where the input $u=u_{\min }=-u_{\max }$ is applied. $x_{m}$ starts decreasing and at $x_{m}=L$, the mode switches to $\left(\right.$ slip $\left._{-}, q_{c}=3\right)$ where $u=u_{\max }$ is applied. This switch is done to reduce the relative velocity, $v_{m}-v_{M}$, back to zero in a shorter time. Once $v_{m}=v_{M}$ the system switches back to $\left(s t i c k, q_{c}=1\right)$ completing the cycle.

Depending on the sign of $\nu$, two cycles are possible, $q_{c}=1-2-3-1$ when $\nu \geq 0$ and $q_{c}=1-4-5-1$ when $\nu \leq 0$. Only the first one will be considered here.

\section{Dehybridization}

The convex combination of the vector fields in the three modes is given by:

$$
\begin{gathered}
\left(\begin{array}{c}
\dot{x}_{m} \\
\dot{v}_{m} \\
\dot{x}_{M} \\
\dot{v}_{M}
\end{array}\right)=\alpha_{1}\left(\begin{array}{c}
v_{m} \\
\frac{\nu}{m+M} \\
v_{M} \\
\frac{\nu}{m+M}
\end{array}\right)+\alpha_{2}\left(\begin{array}{c}
v_{m} \\
\frac{-u_{\max }-k x_{m}+\mathcal{F}_{c}}{m} \\
v_{M} \\
-\frac{\mathcal{F}_{c}}{M}
\end{array}\right) \\
+\alpha_{3}\left(\begin{array}{c}
\frac{v_{m}}{\frac{u_{\max }-k x_{m}+\mathcal{F}_{c}}{m}} \\
v_{M} \\
-\frac{\mathcal{F}_{c}}{M}
\end{array}\right)
\end{gathered}
$$

An upper bound on $\epsilon=\left(\alpha_{2}+\alpha_{3}\right) T$ can be computed, considering the oscillatory behavior of the small mass dynamics and switching condition in mode slip $_{-}$, i.e., $\left(\alpha_{2}+\alpha_{3}\right) T \leq \pi \sqrt{\frac{m}{k}}$. As $(m / k) \rightarrow 0,\left(\alpha_{2}+\alpha_{3}\right) T=$ $\left(1-\alpha_{1}\right) T \rightarrow 0$.

As $(m / k) \rightarrow 0$, the dynamics of the first two states corresponds to the fast dynamics and can be eliminated by singular perturbation. However, by the choice of the new input variable $\nu=u-k x_{m}$ and the switching logic, the position and velocity of the small mass do not enter the dynamics of $x_{M}$ and $v_{M}$ and so, can be simply dropped. The slow dynamics now read:

$$
\left(\begin{array}{c}
\dot{x}_{M} \\
\dot{v}_{M}
\end{array}\right)=\left(\begin{array}{c}
v_{M} \\
-\left(\alpha_{1}-1\right) \frac{\mathcal{F}_{c}}{M}+\frac{1}{m+M} \alpha_{1} \nu
\end{array}\right)
$$

A closed form expression for $\alpha_{1}$ can be obtained in this case: $\alpha_{1}\left(v_{M}, \nu\right)=\frac{-v_{M}+\sqrt{v_{M}^{2}+a \nu}}{-v_{M}+\sqrt{v_{M}^{2}+a \nu}+b \delta \nu}$ with $a, b$, and $\delta$ are appropriate constants.

\section{The continuous control for the averaged model}

Stabilization of the set-point can be done based on the feedback linearization of the averaged nonlinear system. Consider the output dynamics of the nonlinear averaged system (15). This can be linearized via the state feedback control:

$$
\nu=\frac{m+M}{\alpha_{1}}\left(\frac{\alpha_{1}-1}{M} \mathcal{F}_{c}+\nu^{\prime}\right)
$$

to obtain a double integrator system $\ddot{x}_{M}=\nu^{\prime}$. A state feedback controller $\nu^{\prime}=-K x=-k_{p}\left(x_{M}-r\right)-k_{v} v_{M}$ can be designed, for example by a pole placement procedure. The overall control for the inertial drive can be written as:

$$
\begin{gathered}
u=\left\{\begin{array}{ccc}
k x_{m}+\nu & \text { if } & q_{c}=1 \\
-u_{\max } & \text { if } & q_{c}=2,5 \\
u_{\max } & \text { if } & q_{c}=3,4
\end{array}\right. \\
\nu=\frac{m+M}{\alpha_{1}}\left(\frac{\alpha_{1}-1}{M} \mathcal{F}_{c}-k_{p}\left(x_{M}-r\right)-k_{v} v_{M}\right)
\end{gathered}
$$

\subsection{Simulation Results}

In this section, the simulation results for the stickslip inertial drive model is presented. Both the hybrid model and the averaged model are simulated and the results are compared. The numerical values used in the simulation are: $M=1[\mathrm{Kg}], m=0.05[\mathrm{Kg}], \mathcal{F}_{c}=14[\mathrm{~N}]$ and $k=1.76 \times 10^{7}[\mathrm{~N} / \mathrm{m}]$.

For stabilization of the set-point $\left(r=10^{-4}[\mathrm{~m}]\right)$, the control law (17)-(18) with $k_{p}=2 \times 10^{6}$ and $k_{v}=8 \times$ $10^{3}$ is applied. The control gains are found by a pole placement procedure for the feedback linearized model. The control law $\nu$ is applied to the averaged model and the input $u$ to the stick-slip hybrid model. The states of the system for the hybrid model as well as the averaged model is shown in Figure 5. Figure 6 shows the input $u$ applied to the inertial drive $\mathcal{H}_{s}$.

The results show that the behavior of the averaged model is quite close to that of the hybrid system. Also, it can be seen that the proposed methodology stabilizes the system since the approximation error is small. 

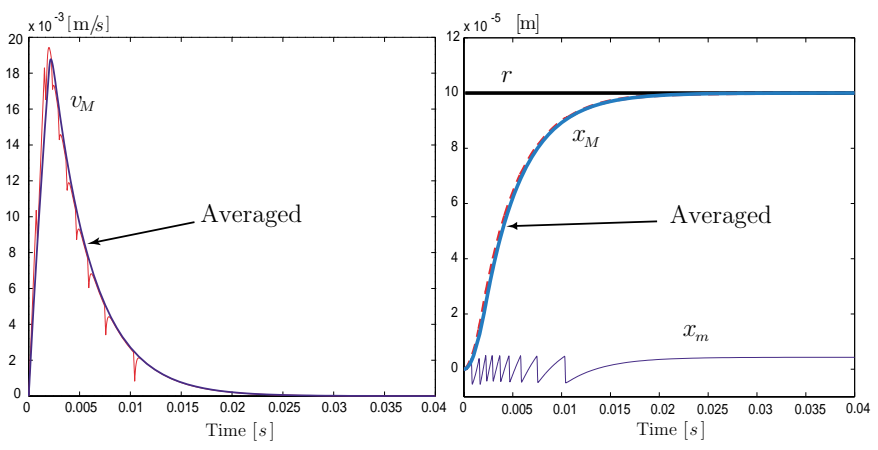

Figure 5: Close loop response of the inertial mass position and velocity for averaged and hybrid models.

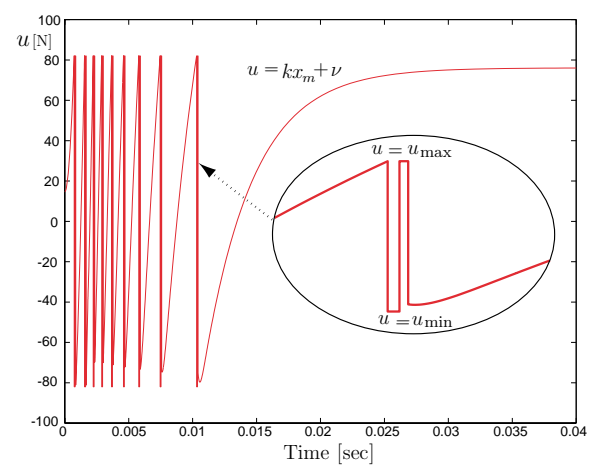

Figure 6: Input $u$ applied to the hybrid model.

\section{Conclusion}

The main idea of this paper is dehybridization - a methodology that approximates a hybrid system by a continuous model. For dehybridization, a preparatory step that ensures the existence of a repetitive mode is necessary. Once a continuous model is obtained from dehybridization, standard techniques can be used for the design of the continuous controller. If the approximation error is sufficiently small, then the proposed controller methodology will stabilize the hybrid system. This methodology was applied in simulation to a stick-slip inertial drive, with encouraging results.

Dehybridization, as presented here, is at a conceptual level and its detailed analysis will be undertaken in the future. Some of the questions that need to be answered are: (i) what class of hybrid systems can be rendered repetitive by an additional switching controller? (ii) how can the approximation error be reduced? and (iii) under what conditions will the overall control strategy be stable?

\section{References}

[1] Bemporad, A., Morari, M., " Control of Systems Integrating Logic, Dynamics, and Constraints." Automatica,,
Vol. 35, pp. 407-428, (1999).

[2] Branicky, S., Borkar, S., Mitter, S.K., "A Unified Framework for Hybrid Control: Model and Optimal Control Theory", IEEE Transaction on Automatic Control, Vol. 43, No.1, pp. 31-45, (1998).

[3] Caines, P.E., Wei, Y.J., "Hierarchical Hybrid Control Systems: A Lattice Theoretic Formulation", IEEE Transaction on Automatic Control, Vol. 43, pp. 501-508, (1998).

[4] Darby, A. P., Pellegrino, S., "Inertial Stick-Slip Actuator for Active Control of Shape and Vibration", Journal of Intelligent Material Systems and Structures, Vol. 8, No.12, pp. 1001-1011, (1997).

[5] DeCarlo, R. A., Branicky, S., Pettersson S., Lennartson, B., "Perspectives and Results on the Stability and Stabilizability of Hybrid Systems", Proceedings of the IEEE, Vol. 88, No.7, pp. 1069-1082, (2000).

[6] Hespanha, J. P., Morse A. S., " Stabilization of Nonholonomic Integrators via Logic-based Switching." Automatica,, Vol. 35, pp. 385-393, (1999).

[7] Karnopp, D., "Computer Simulation of Stick-Slip Friction in Mechanical Dynamic Systems", Transaction ASME Journal of Dynamic Systems, Measurement, and Control, Vol. 107, No.1, pp. 100-103, (1985).

[8] Khalil, H. K., "Nonlinear Systems", Macmillan Co. New York, (1996).

[9] Kotsoukos, X. D., Antsakils, P. J., Stiver, J. A., Lemmon, M. D., "Supervisory Control of Hybrid Systems", Proceedings of the IEEE, Vol. 88, Issue 7, pp. 1026-1049, (2000).

[10] Lygeros, J., Johansson, K.H., Sastry, S., Egerstedt, M., "On the Existence of Executions of Hybrid Automata.", IEEE Conference on Decision and Control, Phoenix, AZ, pp. 2249-2254, (1999).

[11] Michel, A. N., Hu, B., " Towards a Stability Theory of General Hybrid Dynamical Systems." Automatica,, Vol. 35, pp. 371-384, (1999).

[12] Sira-Ramirez, H., Lischinsky-Arenas, P., "Dynamical Discontinuous Feedback Control of Nonlinear Systems.", IEEE Transaction on Automatic Control, Vol. 35, No.12, pp. 1373-1378, (1990).

[13] Srinivasan, B., Sedghi, B., Bonvin, D., "Characterization of the Optimal Solution for A Class of Hybrid Systems." Proceedings of ECC 2001, Porto, Portugal, pp. 1455-1460 (2001).

[14] Tomlin, C.J, Lygeros, J., Sastry, S., " A Game Theoretic Approach to Controller Design for Hybrid Systems.", Proceedings of the IEEE , Vol. 88, No. 7, pp. $949-970$, (2000).

[15] Van der Schaft, A. J., Schumacher, J. M., " The Complementarity Modeling of Hybrid Systems." IEEE Transaction on Automatic Control, Vol. 43, pp. 483-490, (1998). 